\section{MSA Tutorials Videos}

\author{
Greg Erdos \\ University of Florida, Retired, Micanopy, Florida \\ gwe@ufl.edu
}

The Microscopy Society of America, through its Education Committee, has gathered, produced, and distributed video recordings on microscopy and related topics since 1976 . Since 1982, most new acquisitions have been tutorial lectures at MSA Annual Meetings and it is the Society's continuing practice to record all such tutorials for inclusion in the collection. At the same time, MSA continues to augment the Video Library by soliciting relevant titles from a variety of sources. Anyone having, or knowing of, a tape that might be suitable is encouraged to contact the current Chairperson of the Education Committee.

The current catalog is presented below, however, anyone in the future, interested in ordering or purchasing a DVD should refer to the MSA website for the most up to date version of the catalog. (http://www.msa.microscopy.org/MSAUnits/Education/ VideoCatalogue.html). All titles are now available as DVD only. Many of the videos are old and may be of poor technical quality but often contain good information presented by notable microscopists. Please be aware of this when ordering. You will notice that there are gaps in the numbering of videos. These represent lost or corrupted masters. If anyone has a copy of any of the missing numbers, MSA would appreciate borrowing these so that they may be restored to the collection. The title numbers are not in chronological order.

The tutorial organizers are always open to suggestions for new tutorials or recommendations to update past presentations. If you would be willing to present a tutorial or have suggestions in this regard, you may contact the Chairperson of the Education Committee.

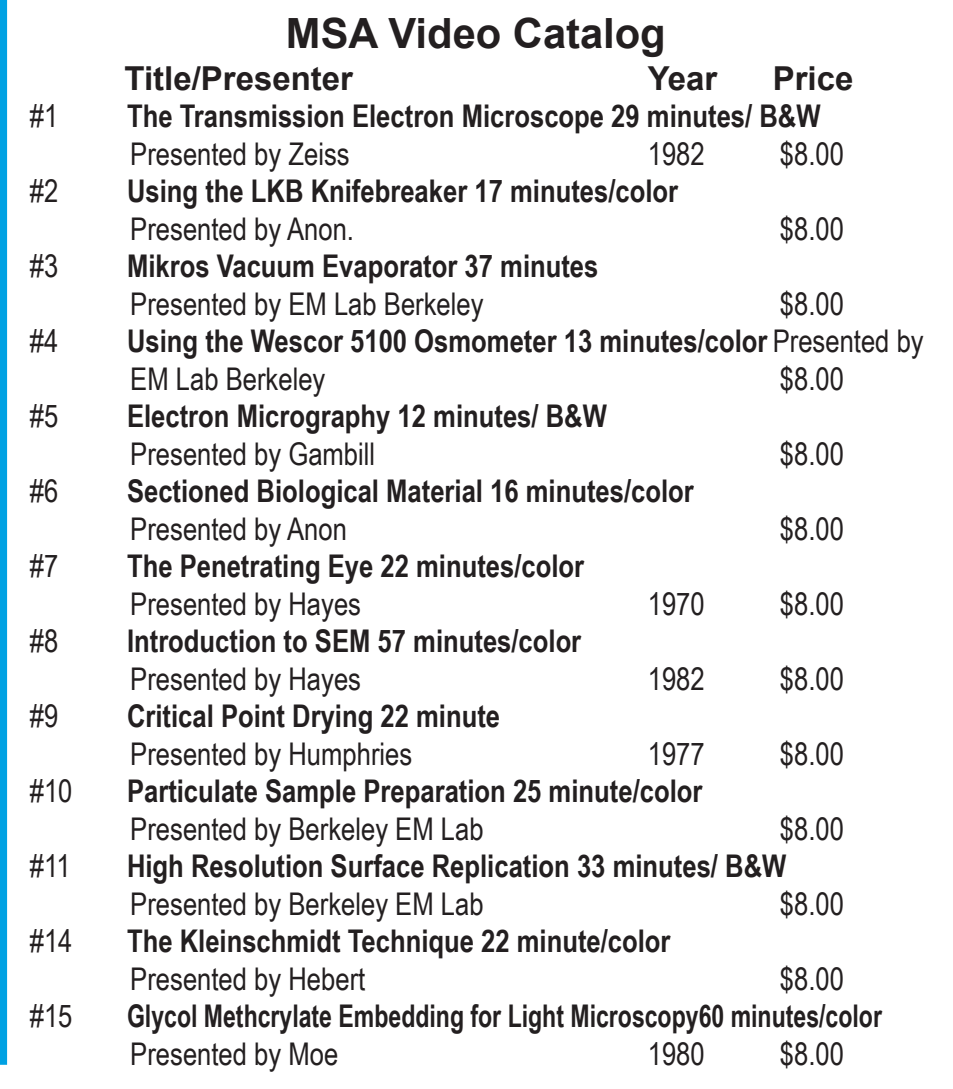

\#16 Interpreting TEM's Three Dimensionally 6 minutes/B\&W

Presented by Pederson

$\$ 8.00$

\#17 Introduction to Freeze-Fracture 77 minutes/color

Presented by Schooley 1982

$\$ 8.00$

\#19 Weak-Beam EM 47 Minutes/color

Presented by VanderSande $\quad 1977 \quad \$ 8.00$

\#20 A lecture on Electron Channeling 47 minutes/color

Presented by Davidson $1978 \$ 8.00$

\#21 Preparation of Macromolecules for TEM 47minutes/color

Presented by Slayter

$\$ 8.00$

\#22 Preparation of Support Films for TEM 14 minutes/color

Presented by Pechak $\quad 1980 \quad \$ 8.00$

\#23 Basic Optics in SEM 40 minutes/ color

Presented by Crang $\quad 1980 \quad \$ 8.00$

\#24 Biological Procedures in EM 41 minutes/ B\&W

Presented by Crang $1971 \quad \$ 8.00$

\#26 Electron Microscopy. Principles and Practice 153 minutes/ B\&W

Presented by Crang $1975 \quad \$ 8.00$

\#27 Operation of the JEOL $100 \mathrm{C} / \mathrm{CX}$ TEM 75 minutes

Presented by Cummings

$\$ 8.00$

\#28 Stereology 52 minutes/color

Presented by Scales $\quad 1982 \quad \$ 8.00$

\#29 JEOL JSM-35 SEM Part I 36 minutes/color

Presented by Thurston

$\$ 8.00$

\#30 JEOL JMS-35 SEM Part II 35 minutes/color

Presented by Thurston

$1978 \$ 8.00$

\#31 Fine Tuning Your SEM 56 minutes/color

Presented by Gaugler

$1982 \$ 8.00$

\#34 Theory of HVEM II 58 minutes/ B\&W

Presented by Humphreys

$1982 \$ 8.00$

\#35 Theory of HVEM III 40 minutes/ B\&W

Presented by Humphreys

$1982 \$ 8.00$

\#36 Theory of HVEM IV 57 minutes/ B\&W

Presented by Humphreys

$1982 \$ 8.00$

\#37 Kinetic Studies I 57 minutes/ B\&W

Presented by Loretto

\#38 Kinetic Studies II 44 minutes/ B\&W

$1982 \$ 8.00$

Presented by Loretto

$1982 \$ 8.00$

\#39 Kinetic Studies III 74 minutes/ B\&W

Presented by Westmacott:

\#40 Gas-Solid Interactions

Presented by Kenik:

\#41 Radiation Damage I 96 minutes/ B\&W

Presented by Kiritani

Radiation Damage III 50 minutes/ B\&W

Presented by Merkle

\#44 Radiation Damage IV 22 minutes/ B\&W

Presented by King

Introduction to Ultramicrotomy 36 minutes/color

Presented by Schooley 1982

\#46 Colloidal Gold Labeling 112 minutes

Presented by DeMee 1983

\#48 Glass \& Ceramics; lon Milling 90 minutes/color

Presented by Howitt 1983

\#53 Basics of STEM 50 minutes/color

Presented by VanderSande:

$\$ 8.00$

$\$ 8.00$

1982

$\$ 8.00$

How to Read a Converd

$\$ 8.00$

Presented by Eades

\#55

\#57 High Resolution EM 55 minutes/ color

Presented by Gibson

1982

$\$ 8.00$

$\$ 8.00$

$\$ 8.00$

$\$ 8.00$

$\$ 8.00$

\#58 


\section{Side-By-Side Comparison? Difficult When Our Coaters Stand Alone.}

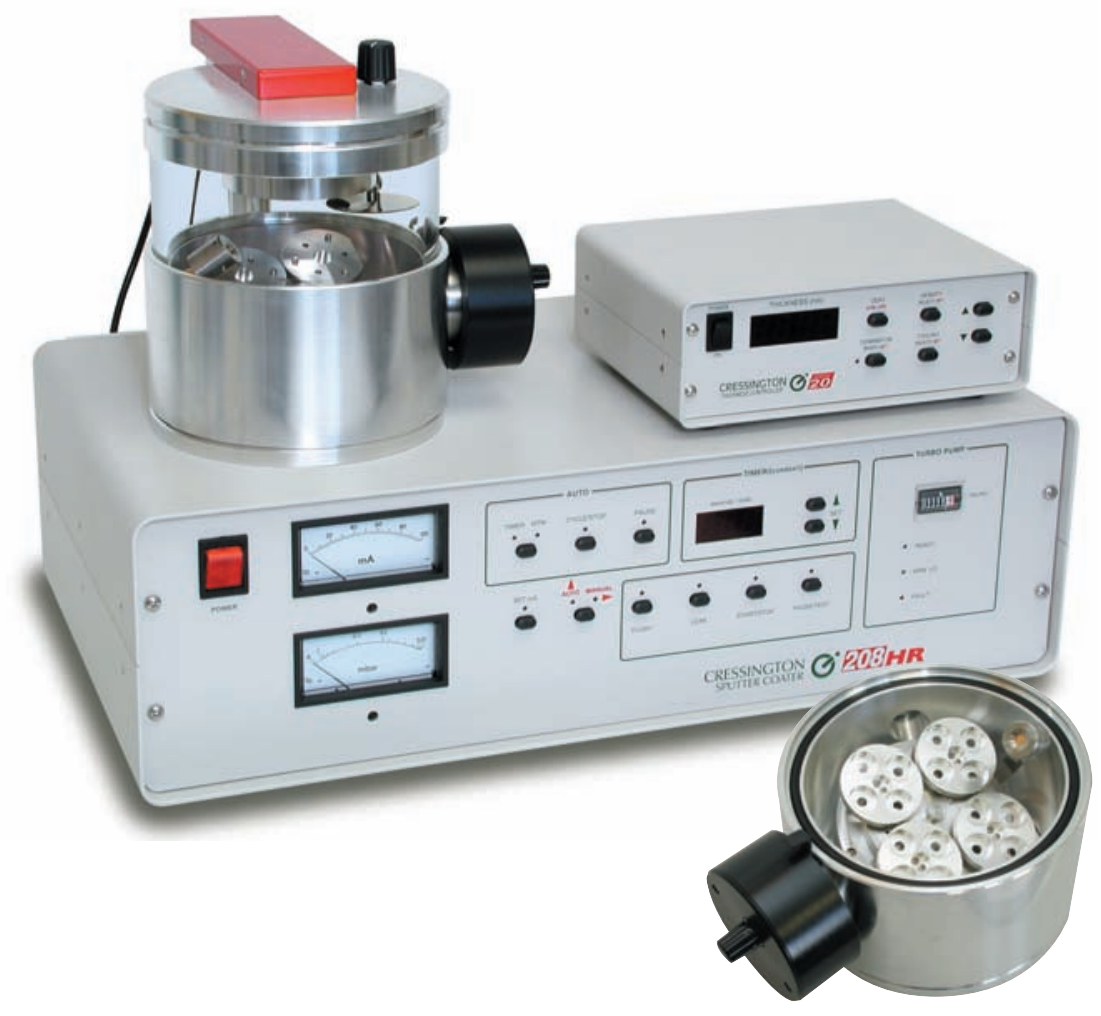

\section{High Resolution Sputter Coater}

\section{HR for FE-SEM}

\section{Superior Features:}

- High Resolution Fine Coating

- Wide Choice of Coating Materials

- High Resolution Thickness Control

- Multiple Sample Stage Movements

- Wide Range of Operating Pressures

- Compact, Modern, Benchtop Design
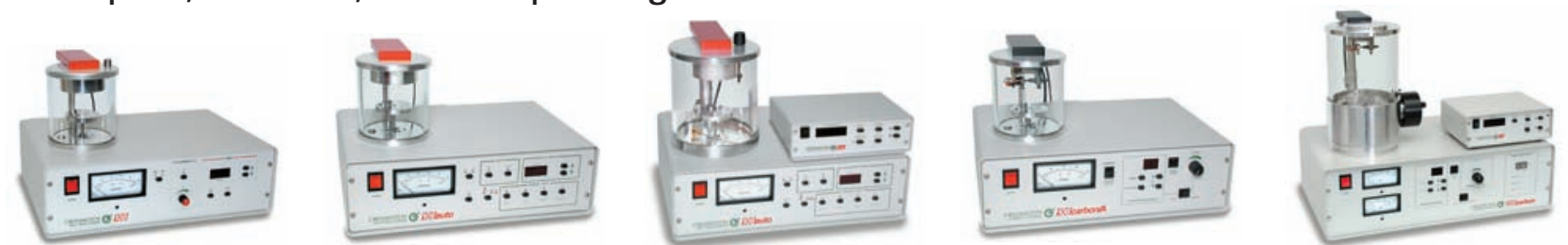

Find out about our complete line of sample coaters. 


\begin{tabular}{|c|c|c|c|}
\hline \multirow{4}{*}{$\begin{array}{l}\# 59 \\
\# 60\end{array}$} & \multicolumn{3}{|c|}{ Additives in Biological Fixation 76 minutes/color } \\
\hline & Presented by Boyles & 1984 & $\$ 8.00$ \\
\hline & \multicolumn{3}{|l|}{ Rapid Freezing of Biological Specimens } \\
\hline & \multicolumn{3}{|l|}{ Presented by Costello } \\
\hline \multirow[t]{2}{*}{ \#61 } & \multicolumn{3}{|l|}{ Cryoultramicrotomy 54 minutes/ color } \\
\hline & Presented by Hagler & 1984 & $\$ 8.00$ \\
\hline \multirow[t]{2}{*}{ \#62 } & \multicolumn{3}{|c|}{ Image Recording and processing 30 minutes/color } \\
\hline & Presented by Krakow & & $\$ 8.00$ \\
\hline \multirow[t]{2}{*}{ \#63 } & \multicolumn{3}{|l|}{ HVEM for Biologists 50 minutes } \\
\hline & Presented by Gronsky & 1984 & $\$ 8.00$ \\
\hline \multirow[t]{2}{*}{ \#67 } & \multicolumn{3}{|l|}{ Low Dose Imaging 63 minutes/color } \\
\hline & Presented by Downing & 1985 & $\$ 8.00$ \\
\hline \multirow[t]{2}{*}{ \#71 } & \multicolumn{3}{|c|}{ Specimen Preparation for Near Surface Examination 48 minutes/colo } \\
\hline & Presented by Horton & 1985 & $\$ 8.00$ \\
\hline$\# 72$ & \multicolumn{3}{|c|}{ Immunocytochemical Localization 49 minutes/color } \\
\hline & Presented by Pickel & 1985 & $\$ 8.00$ \\
\hline \#73 & Energy Dispersive X-Ray Analysis $90 \mathrm{mir}$ & utes/co & \\
\hline & Presented by Fiori & 1985 & $\$ 8.00$ \\
\hline$\# 74$ & Balzers 301 Freeze-Fracture Apparatus 5 & minute & olor \\
\hline & Presented by Rash & & $\$ 8.00$ \\
\hline$\# 76$ & Ultrarapid Propane Jet Freezing 120 min & Ites & \\
\hline & Presented by Gilkey & 1986 & $\$ 8.00$ \\
\hline$\# 77$ & Biological Applications of Cryo-SEM 57m & inutes/c & \\
\hline & Presented by Sargent & 1986 & $\$ 8.00$ \\
\hline \#79 & Post-Shadow Labeling 40 minutes & & \\
\hline & Presented by Dinduk & 1986 & $\$ 8.00$ \\
\hline \#83 & X-Ray Microanalysis 60 minutes/color & & \\
\hline & Presented by Fiori & 1986 & $\$ 8.00$ \\
\hline \#84 & LaB6 in the TEM & & \\
\hline & Presented by Sewell & & $\$ 8.00$ \\
\hline \#87 & High Resolution SEM & & \\
\hline & Presented by Peters & 1986 & $\$ 8.00$ \\
\hline$\# 90$ & CBED Total of 3 tapes 280 minutes & & \\
\hline & Presented b & 1986 & $\$ 8.00$ \\
\hline$\# 91$ & CBED Approx. 300 minutes & & \\
\hline & Presented by Steeds & 1986 & $\$ 8.00$ \\
\hline$\# 92$ & CBED Approx. 280 minutes & & \\
\hline & Presented by Spence & 1986 & $\$ 8.00$ \\
\hline \#93 & CBED & & \\
\hline & Presented by Bird & & $\$ 8.00$ \\
\hline \#94 & Nuclear Microprobe Analysis 62 minutes & color & \\
\hline & Presented by Doyle & 1986 & $\$ 8.00$ \\
\hline$\# 98$ & Development of Beam-forming System 2 & minute & olor \\
\hline & Presented by Mulvey & 1986 & $\$ 8.00$ \\
\hline \#103 & Microanalysis B.C.31 minutes/color & & \\
\hline & Presented by Heidenreich & 1986 & $\$ 8.00$ \\
\hline \#105 & Basic Optics in TEM & & \\
\hline & Presented by McConville: & & $\$ 8.00$ \\
\hline \#106 & Electron Scattering & & \\
\hline & Presented by VanderSande & & $\$ 8.00$ \\
\hline \#107 & Contrast Mechanisms in TEM 57 minutes & color & \\
\hline & Presented by Hren & 1987 & $\$ 8.00$ \\
\hline \#109 & Care and Use of Diamond Knives 56 min & Ites/colc & \\
\hline & Presented by Bell & 1987 & $\$ 8.00$ \\
\hline$\# 110$ & EM of Nucleic Acids 47 minutes/color & & \\
\hline & Presented by Beer/Varkey & 1987 & $\$ 8.00$ \\
\hline \#112 & Preparation of Thin Foils for TEM 59 min & tes/colc & \\
\hline & Presented by Goodhew & 1987 & $\$ 8.00$ \\
\hline \#113 & Image Recording in the EM 45 minutes/c & & \\
\hline & Presented by Black: & 1987 & $\$ 8.00$ \\
\hline \#114 & $\begin{array}{l}\text { Making Every Electron Count. Detectors } \\
\text { color }\end{array}$ & or SEM & minutes/ \\
\hline & Presented by McMullen & 1987 & $\$ 8.00$ \\
\hline \#115 & Interview 29 minutes/color & & \\
\hline & Presented by LePoole/Zeitler & 1987 & $\$ 8.00$ \\
\hline
\end{tabular}

\#116 Unknown Phases by ED/EDS 58 minutes.

$\begin{array}{lll}\text { Presented by Carr } & 1988 & \$ 8.00\end{array}$

\#117 Planar Interfaces \& Defects 55 minutes/color

Presented by Pond: $\quad 1988 \quad \$ 8.00$

\#118 TEM Characterization of Precipitates 49 minutes/color.

Presented by Dahmen $1988 \quad \$ 8.00$

\#121 Confocal Scanning Light Microscopy 63 minutes/color.

Presented by Boyde $\quad 1988 \quad \$ 8.00$

\#127 High Spatial Resolution Microanalysis in the AEM 84 minutes

Presented by Garrett-Reed $\quad 1989 \quad \$ 8.00$

\#130 Colloidal Gold 18 minutes.

Presented by Albrecht/Simmons $\quad 1990 \quad \$ 8.00$

\#132 Scanning Tunneling Microscopy

Presented by Chiang $\quad 1990 \quad \$ 8.00$

\#135 Quantitative Image Analysis 64 minutes.

Presented by Russ $\quad 1990 \quad \$ 8.00$

\#136 Convergent Beam Electron Diffraction 51 minutes

$\begin{array}{lll}\text { Presented by Eaglesham } & 1990 \quad \$ 8.00\end{array}$

\#137 Ultramicrotomy 50 minutes.

Presented by Leica $\quad 1990 \quad \$ 8.00$

\#138 Sectioning for Serial Reconstruction $\mathbf{4 0}$ min

Presented by Kinnamon $\quad 1991 \quad \$ 8.00$

\#140 3-D IVEM Tomography

Presented by Agard $\$ 8.00$

\#144 EM of Ceramics

Presented by Mitchell $\quad 1991 \quad \$ 8.00$

\#146 Confocal LM for Biology

Presented by Shuman $\quad 1991 \quad \$ 8.00$

\#147 Freeze Fracture of Membranes

Presented by Zampighi $\$ 8.00$

\#148 Fundamentals of ALCHEMI

Presented by Turner $\quad 1991 \quad \$ 8.00$

\#151 Fluorescence in situ Hybridization in Biomedical Research

$\begin{array}{lll}\text { Presented by J. Jerome } & 1992 & \$ 8.00\end{array}$

\#152 Atomic Force Microscopy

Presented by Fisher

\#153 High Resolution MA

Presented by Romig

$1992 \$ 8.00$

\#158 Failure Analysis Methods

Presented by Anderson $\quad 1992 \$ 8.00$

\#159 Biometrics

Presented by Samakaya $\quad \$ 8.00$

\#160 Cryopreservation

Presented by Costello $\quad 1993 \quad \$ 8.00$

\#161 SIMS

Presented by Linton $\quad 1993 \quad \$ 8.00$

\#162 Electron Excited x-ray Microanalysis

Presented by Newbury

$\$ 8.00$

\#163 Microstructure Characterization

Presented by Black

$1993 \$ 8.00$

\#164 Introduction to the Forgotten Art of Electron Diffraction Contrast

Presented by D. Maher $\quad 1993 \quad \$ 8.00$

\#166 Computer-aided Analytic \& Visualizations Tools for Structural Studies

Presented by B. Carragher $\quad 1993 \quad \$ 8.00$

\#168 Recent Advances in Light Microscopy

Presented by B. Herman $\$ 8.00$

\#170 A Basic Introduction to Image Processing Using NIH Image Presented by J. Mansfield $1995 \quad \$ 8.00$

\#179 A Few Words on Bits and Bytes: A Tutorial on Image Spectral Processing for the Novice 120 Minutes

Presented by J.F. Mansfield $1995 \quad \$ 8.00$

\#181 Atom-Probe Analysis of the Solid-State Liquid Interface $120 \mathrm{~min}$. Presented by J.A. Panitz $\quad 1995 \quad \$ 8.00$

\#182 Image processing and analysis fundamentals for microscopy 120 minutes

Presented by B.D.Newell

1995

$\$ 8.00$ 


\section{Beating the Competition is Easy, When You've Got Products They Don't Offer!}

\section{Si-Li EDS Detectors,}

IXRF offers guaranteed, premium $\leq \underline{130 \mathrm{eV}}$, resolution Si-Li detectors. (*industry standard detector resolution $133-138 \mathrm{eV}$ ) that carry a three year warranty.

New $30 \mathrm{~mm}^{2}$ at $133 \mathrm{eV}$, three year warranty available for cost effective fast X-ray Mapping.

\section{fX SEM XRF,}

400-1000 micron spot. 10,000

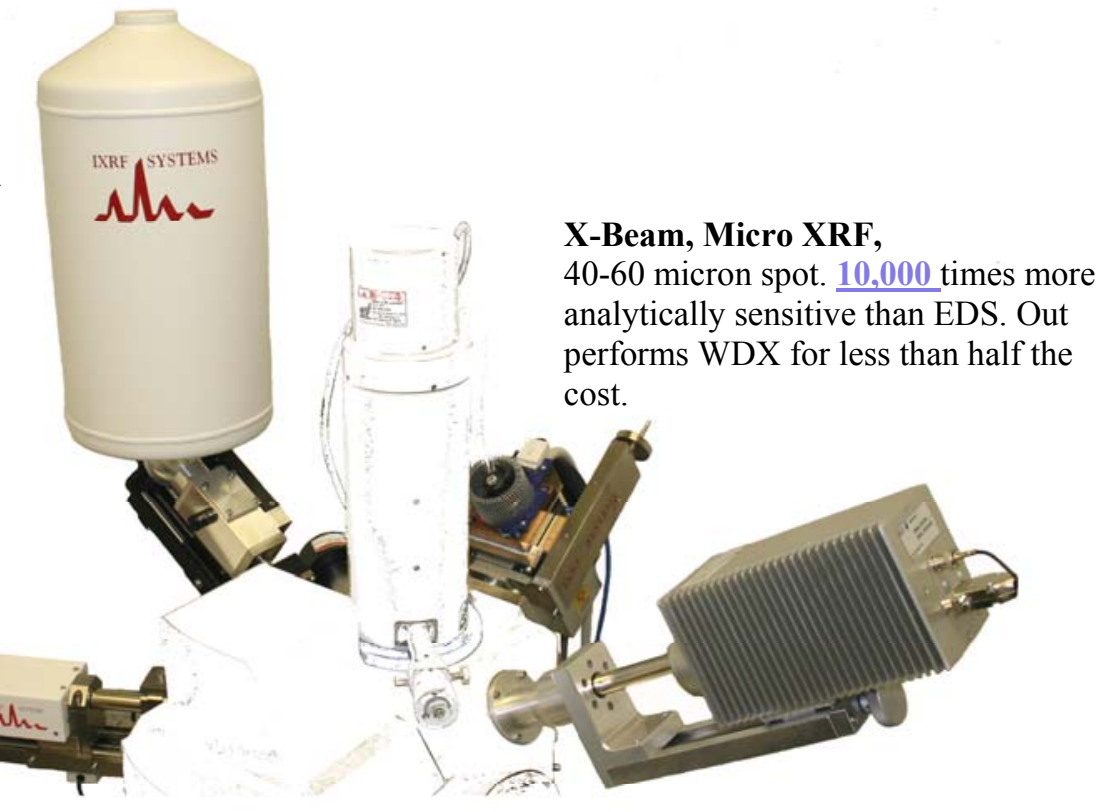

times more analytically sensitive than EDS.

Perfect for environmental applications as well as non-conductive samples.
50 $\mathbf{m m}^{2}$ Silicon Drift Detector, the largest Active Area in the industry $\left(50 \mathrm{~mm}^{2}\right) .133 \mathrm{eV}$ resolution, with optimum peak stability, and high input count rates. Who says "Bigger isn't better"?

IXRF does not stratify their software suite into low, medium, and high-end levels; there's only one high-end package that includes a myriad of Spectra Analysis, Image Analysis, X-ray

Mapping, and unsurpassed SEM/EDS Automation.

Free Software Upgrades for Life...no compromises. 
\#183 3-D microscopy, confocal, deconvolution or both 90 minutes

Presented by J.B. Pawley $1996 \quad \$ 8.00$

\#184 Ion beam milling materials with applications to TEM specimen preparation 90 minutes

Presented by R. Anderson

$1996 \$ 8.00$

\#185 Five dimensional microscopy using wide field deconvolution, Practical considerations and biological applications $\mathbf{5 0}$ minutes Presented by W.F. Marshall $\quad 1996 \quad \$ 8.00$

\#186 3-D Microscopy using confocal microscopy. 50 minutes, Presented by E.H.K. Stelzer $\quad 1996 \quad \$ 8.00$

\#197 Negative staining: a valuable technique for studying subcellular components

Presented by S.E. Miller $\quad 1997 \quad \$ 8.00$

\#198 Gamma Correction

Presented by J. MacKenzie $\quad 1997 \quad \$ 8.00$

\#199 Freeze fracture at the turn of the Century: techniques for visualizing and labeling tissues,cells and molecules

Presented by Yasamura \& Rash $\quad 1997 \quad \$ 8.00$

\#200 Focussed ion beam milling for site specific TEM

Presented by L.A. Gianuzzi, J.L. Drown, S.R. Brown,R.B. Erwin

\& F.A. Stevie $\quad 1997 \quad \$ 15.00$

\#201 Practical Methods for TEM

Presented by K. Chien, R. Gonzalez, R. Heusser, H. Shiroishi \& M.Heatherstraw $1998 \quad \$ 15.00$

\#202 Ethics in digital imaging

Presented by S. Silvers, J. Kinnamon, R. Mattson,

S. Dunn $1998 \$ 15.00$

\#203 The development and application of the tripod polishing

Technique

Presented by J. Benedict, S. Klepies and

R. Anderson $1998 \$ 15.00$

\#204 Tech. Forum, Instrumentation: How to choose it and use it . SPM and SEM

Presented by Russel \& Lamberti $\quad 1998 \quad \$ 15.00$

\#205 Tech Forum Instrumentation how mto choose it and use it.

Designing a microscopy lab

Presented by Murphy $1998 \quad \$ 15.00$

\#206 Tech Forum Instrumentation: How to choose it and use it.

Microtomes and associatedequipment. Confocals

Presented by Bozzola \& Drazba $\quad 1998 \quad \$ 15.00$

\#207 Tech. Roundtable: Photoshop 101

Presented by Multiple $\quad 1998 \quad \$ 15.00$

\#208 Outstanding Technologists Awards Recipients.

Presented by Multiple $\quad 1998 \quad \$ 15.00$

\#209 Miniaturized Artificial Machines in Biology

Presented by Multiple Speakers $\quad 1998 \quad \$ 15.00$

\#210 25 Years in a Stereology Laboratory: a Point by Point History

Presented by John Basgen $\quad 1999 \quad \$ 8.00$

\#211 Transmission electron microscope specimen preparation of metal matrix composites using the focused ion beam miller. 80 minutes Presented by P. Munroe, J. Cairney, R. Smith $1999 \quad \$ 15.00$

\#212 SEM at a distance. Nuts \& Bolts

Presented by S. B. Barlow $\quad 1999 \quad \$ 15.00$

\#213 Factors Influencing Success of an Academic EM Facility Presented by Wheatly $1999 \quad \$ 8.00$

\#214 Utility of Secondary Guard Hairs in Animal Hair Identification Presented by Yates $\quad 1999 \quad \$ 8.00$

\#215 The Basics of Microtomy for Materials Science Microscopy Presented by T. Malis $\quad 1998 \quad \$ 15.00$

\#216 Typical Application in Metalurgical Failure Ananlysis Laboratories Presented by Wong $\quad 1999 \quad \$ 8.00$

\#218 Uses of Microscopy in the Cime Lab

Presented by McAdam $\quad 1999 \quad \$ 8.00$

\#219 Funding in the 90's

Roundtable

$1999 \$ 8.00$

\#220 Detecting \& OptimizingFluorescence Signals

Presented by Metcalf $\quad 1999 \quad \$ 8.00$

\#221 Single Particle Analysis of Macromolecules and Complexes: How to Get Started. 60 Minutes

Presented by S J Ludtke $2000 \$ 15.00$

\#222 Structural Analysis of Proteins on Lipid Substrates. $1 \mathrm{hr}$. Presented by E M Wilson-Kubalek $\quad 2000 \quad \$ 15.00$

\#223 Guide to Three-Dimensional Reconstruction of helical Structures Presented by DeRosier $\quad 2000 \quad \$ 15.00$

\#224 Microscopy \& Microanalysis Over The Net Presented by N J Zaluzec $\quad 2000 \quad \$ 15.00$

\#225 Multi-Photon Excitation Microscopy: An Old Idea in Quantum Theory Applied to Modern Scientific Problems 90 minutes.

Presented by D W Piston $\quad 2000 \quad \$ 15.00$

\#226 Energy Filtered Imaging

Presented by J. Bentley

\#227 Atom Probe Tomography

Presented by M.K. Miller

$2000 \$ 15.00$

Rable: Safety Issues in the Microscopy Lab 60 minutes

Presented by S H Silvers, : E. Boylston, $2000 \quad \$ 15.00$

\#229 Computational and Experimental Methods in Molecular

Microscopy

Presented by NA

$2000 \$ \$ 15.00$

\#233 3-D Visualization Tools

Presented by M.T. Dougherty $\quad 2001 \quad \$ 15.00$

\#234 Selective Staining, Contrast Enhancement for biological electron tomography

Presented by M. H. Ellisman $2001 \quad \$ 15.00$

\#236 Topics in Electron Diffraction TEM

Presented by J.A. Eades $\quad 2001 \quad \$ 15.00$

\#237 Running a multipurpose Microscopy Laboratory

Presented by C.E. Nockolds 2001

\#238 Variable Pressure SEM. How We image with Them

Presented by B.J. Griffin.

$2001 \$ \$ 15.00$

\#239 Low Voltage SEM (2 tapes)

Presented by David Joy \& Donald Newbury $2001 \quad \$ 15.00$

\#240 ESEM/LV/VP: Imaging at Low Vacuum

Presented by Symposium $\quad 2001 \quad \$ 15.00$

\#241 Dual Beam Instrumentation

Presented by L.A. Giannuzzi

$2001 \$ \$ 15.00$

\#242 Remote Microscopy in Shared and Teaching Facilities

Presented by Roundtable. $\quad 2001 \quad \$ 15.00$

\#243 Spectral Imaging

Presented by P.T. Kotula \& M.R. Keenan $2002 \quad \$ 15.00$

\#248 Scanned Probe Microscopy

Presented by P. Russell

$2002 \$ 15.00$

\#250 Basic Confocal Microscopy - Part I

Presented by J. Jerome \& R. Price

$2002 \$ 15.00$

\#251 Basic Confocal Microscopy - Part II

Presented by J. Jerome \& R. Price

$2002 \$ \$ 15.00$

\#252 TEM \& Polymers

Presented by J.S. Vastenhout

$2002 \$ 15.00$

\#253 Electron Backscatter Detection in SEM

Presented by J.R. Michael

$2002 \$ \$ 15.00$

\#254 Cellular Dynamics using AFM

Presented by T, Lehto, et al.

$2002 \$ 15.00$

\#255 Cryo-TEM of Large Complexes

Presented by S. Fuller

$2002 \$ \$ 15.00$

\#256 Quality Systems for Microscopy \& Microanalyisis

Presented by E. Steele 2002

$\$ 15.00$

\#257 Electron Energy Loss Spectroscopy

Presented by R.F. Egerton

$2003 \$ \$ 15.00$

\#258 Development of CCD Based Digital Imaging for TEM

Presented by S. Peltier \& J. Bouwer $2003 \quad \$ 15.00$

\#259 High Resolution TEM: Tomograohy - Principle \& Practice

Presented by D.J. Smith $\quad 2003 \quad \$ 15.00$ 


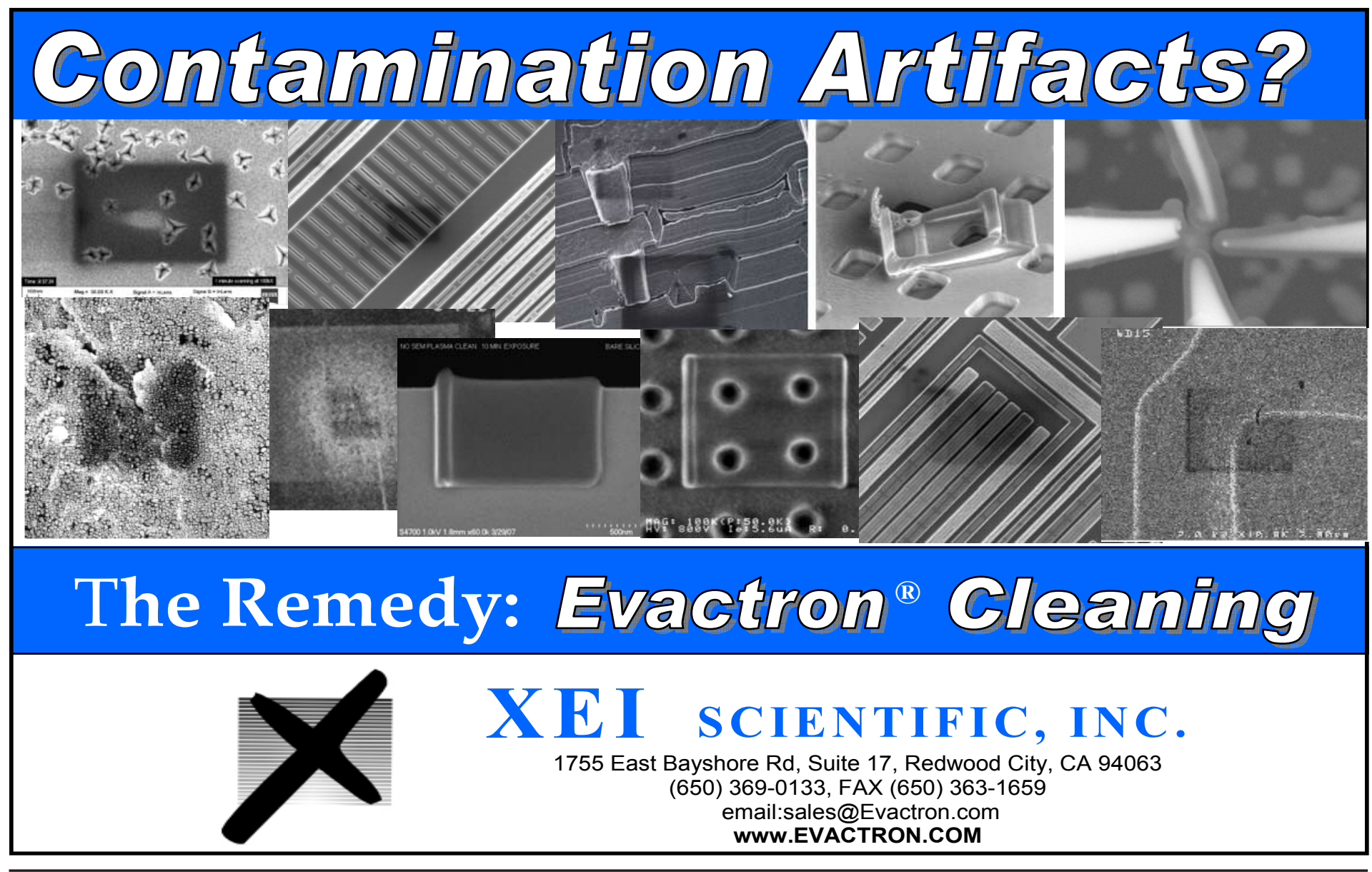

Microanalysis

X-ray detector technology from e2v scientific instruments

e2v

www.e2vsi.com

T: $+44(0) 1628533060$

$\int \Lambda$ T: +1 9145926050

E: e2vsi@e2v.com

E: e2vsi-na@e2v.com

\section{SiriusSD ${ }^{\circledR}$ Silicon drift detector for EDS applications}

With superior analytical quality spectra, the SiriusSD makes light work of EDS analysis.

Our premium quality X-ray detectors offer resolutions down to $126 \mathrm{eV}$ with excellent light element performance. Using the SiriusSD's high rate capability quality phase maps can be recorded in minutes.

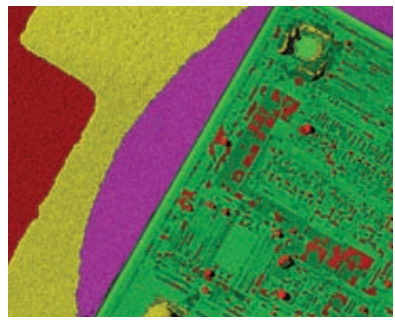

High speed phase mapping

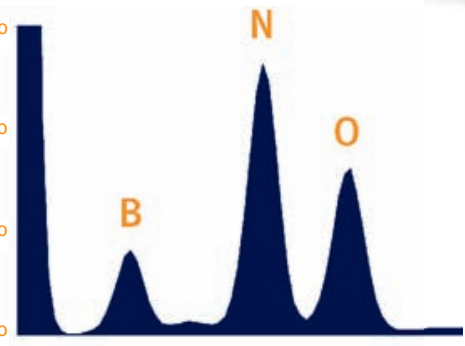

Superior light element performance

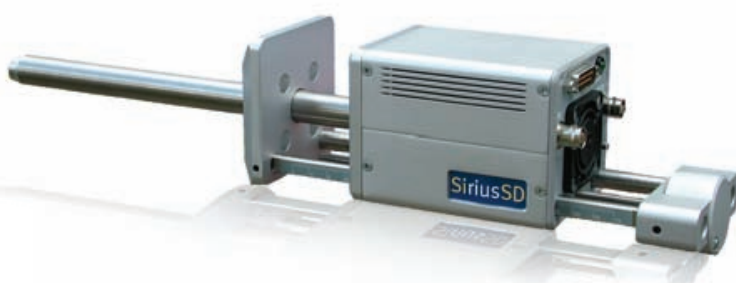

- Resolution from $126 \mathrm{eV}$

- $10 \mathrm{~mm}^{2}$ and $30 \mathrm{~mm}^{2}$ active areas

- Light element and Beryllium windows available 
\#260 Image Databases: What are they \& What do They Bring to Microscopy

Presented by Jose Maria Carazo

$2003 \$ \$ 15.00$

\#261

Creating Site Specific Training Tools

Presented by Steven B. Barlow

$2003 \$ \$ 15.00$

\#262 Introduction to the SEM

Presented by David Joy \& Donald Newbury $2003 \quad \$ 15.00$

\#263 More Basic Confocal Microscopy - Part 1

Presented by J. Jerome \& R. Price

$2003 \$ \$ 15.00$

\#264 More Basic Confocal Microscopy - Part 2

Presented by R. Price \& J. Jerome

\#265 More Basic Confocal Microscopy - Part 3

Presented by J. Jerome \& R. Price
Techniques for Electron Tomography

Presented by M. Marko

$2003 \$ \$ 15.00$

\#266

\#267 Energy Dispersive X-ray spectrometry in SEM

Presented by Dale Newbury

$2003 \$ \$ 15.00$

Introduction to Electron Holography

Presented by Molly McCartney $2004 \quad \$ 15.00$

\#269 Introduction to Fluorescence and Image Correlation

Spectroscopy

Presented by P. Wiseman

$2004 \$ \$ 15.00$

\#270 Practical STEM

Presented by David Muller

$2004 \$ \$ 15.00$

\#271 Building Order in Large Image Data Sets: Classification

Techniques at Work!

Presented by J M Carazo Garcia $\quad 2003 \quad \$ 15.00$

\#272 Computational Tools for Interpreting Electron Tomograms

Presented by N Volkmann

$2005 \$ \$ 15.00$

\#273 Confocal Microscopy System Performance: Spectroscopy and Foundations for Quantitation

Presented by R M Zucker; $\quad 2005 \quad \$ 15.00$

\#274 Improved Electron Microscopy with Monte Carlo Simulations Presented by R Gauvin $2005 \quad \$ 15.00$

\#275 In-situ Electron Microscopy: A Practical Tutorial
Presented by E A Stach
2005
$\$ 15.00$

\#276 Quantum Dots as Cellular Probes for Light and Electron Microscopy Presented by Deerinck,Giepmans,Ellisman $2005 \quad \$ 15.00$

\#277 Theory and Applications of Focal-Series Reconstruction in
HRTEM Presented by A Thust
2005
$\$ 15.00$

\#278 Fluctuation Electron Microscopy for the MASses

Presented by M M J Treacy $2005 \quad \$ 15.00$

\#279 Solve the Mystery (EM for kids)

Presented by Elaine Humphrey $2005 \quad \$ 15.00$

\#280 How to Use the Dual Beam FIB-SEM to Characterize Microsctructure Presented by Micahel Uchic $\quad 2006 \quad \$ 15.00$

\#281 Tomographic Reconstruction with the IMOD Software Package Presented by David Mastronarde $\quad 2006 \quad \$ 15.00$

\#282 Atom Probe Tomography and Applications to Understanding Nanotechnology

Presented by S.P. Ringer $\quad 2006 \quad \$ 15.00$

\#283 CCD Technology for Digital Image Capture on Transmission Electron Microscopes

Presented by Brent Bailey $\quad 2006 \quad \$ 15.00$

\#284 Microwave Processing in a Modern Microscopy Facility

Presented by Elaine Humphry $2006 \quad \$ 15.00$

\#285 How to convert 2-D sectioning images to a 3-D Data Set

Presented by M. De Graef $\quad 2006 \quad \$ 15.00$

\#286 Serial Sectioning in the Miceon Plus Range \& Modern Techniques for Automation

Presented by Jonathan Spowart

$2006 \$ 15.00$

\#287 Cryoelectron Tomography

Presented by Daniella Nicastro

$2006 \$ 15.00$

\#288 Early Times of Electron Probe Analysis

Presented by NA

1986
\#289 Development of the Beam Forming System of the Electron

Microprobe

Presented by NA $\quad 1986 \quad \$ 8.00$

\#291 The many Skills of Microprobe Analysis: Absorption Correction and ZAF Analysis

Presented by NA

$1986 \$ \$ 8.00$

\#292 Round Table Discussion

Presented by Castaing Cosslettt Duncomb Mulvey Phillibert $1986 \$ 8.00$

\#293 Microanalysis B.C.

Presented by NA $\quad 1986 \quad \$ 8.00$

\#294 Formation of the Summer School for x-ray Microanalysis at MIT: Development of Electron Microprobe Instrumentation.

Presented by NA $\quad 1986 \quad \$ 8.00$

\#295 Early Work on Microprobe Analysis in Japan

Presented by Shinoda $\quad 1986 \quad \$ 8.00$

\#296 Biological Electron-Probe X-ray Analyisis Development of

Geological Applications

Presented by NA $\quad 1986 \quad \$ 8.00$

\#297 Round Table Discussion

Presented by Hall Heinrich Kell Wittry Ogilvie Shimizu $1986 \$ 8.00$

\#298 Tutorial: Nuclear Microprobe Analysis

Presented by Sandi Nat'l Labs $\quad 1986 \quad \$ 8.00$

\#299 High pressure freezing for electron microscopy of biological specimens. and Freeze substitution method: tutorial and roundtable discussion

Presented by Stock, Studer, McDonald $\quad 2007 \quad \$ 15.00$

\#300 A novel sample freezing method

Presented by Leunissen $\quad 2007 \quad \$ 15.00$

\#301 Electron tomography for the materials science

Presented by Midgley 2007

\#302 LACSBI: incoherent imaging for the quantitative TEM

Presented by lan Anderson $\quad 2007 \quad \$ 15.00$

\#303 Atomic force microscopy (AFM) and related microscopy

Techniques and applications

Presented by Russell $\quad 2007 \quad \$ 15.00$

\#304 Creating a successful scientific presentation (Professional Development tutorial)

Presented by Bev Maleef $\quad 2007 \quad \$ 15.00$

\#305 Playing the grant game to get the toys (instruments) we want (Professional Development tutorial)

Presented by Price

$2007 \$ 15.00$

\#306 X-ray microCT Instructor: Stuart Stock

Presented by Stock

$2007 \$ \$ 15.00$

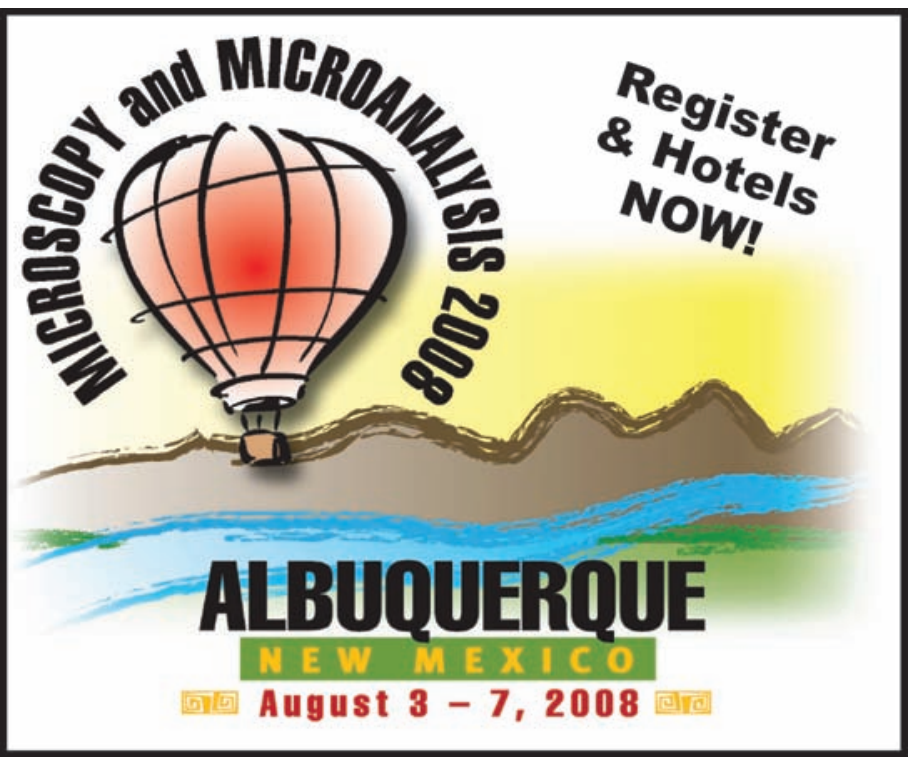

